\title{
INTRINSIC GALAXY ALIGNMENTS AND WEAK GRAVITATIONAL LENSING
}

\author{
A. F. HEAVENS \\ Institute for Astronomy, University of Edinburgh \\ Blackford Hill, Edinburgh EH9 3HJ, UK \\ E-mail: afh@roe.ac.uk
}

\begin{abstract}
Gravitational lensing causes background galaxy images to become aligned, and the statistical characteristics of the image alignments can then be used to constrain the power spectrum of mass fluctuations. Analyses of gravitational lensing assume that intrinsic galaxy alignments are negligible, but if this assumption does not hold, then the interpretation of image alignments will be in error. As gravitational lensing experiments become more ambitious and seek very low-level alignments arising from lensing by large-scale structure, it becomes more important to estimate the level of intrinsic alignment in the galaxy population. In this article, I review the cluster of independent theoretical studies of this issue, as well as the current observational status. Theoretically, the calculation of intrinsic alignments is by no means straightforward, but some consensus has emerged from the existing works, despite each making very different assumptions. This consensus is that

a) intrinsic alignments are a small but non-negligible $(\lesssim 10 \%)$ contaminant of the lensing ellipticity correlation function, for samples with a median redshift $\bar{z} \sim 1$; b) intrinsic alignments dominate the signal for low-redshift samples $(\bar{z} \sim 0.1)$, as expected in the SuperCOSMOS lensing survey and the Sloan Digital Sky Survey.
\end{abstract}

\section{Introduction}

\subsection{Lensing and galaxy shapes}

Galaxy shapes are being used increasingly as a tool in cosmology. Specifically, correlations of the orientations of galaxies on the sky, which arise as a result of gravitational lensing, can be used to measure the mass distribution in the Universe. As cosmology becomes more of a precision science, with accurate estimation of cosmological parameters possible from microwave background observations, weak lensing offers a complementary accurate method of studying the state of the cosmos. Traditional studies of large-scale structure focus on the clustering properties of galaxies, but this approach suffers from the disadvantage that galaxies may not be unbiased tracers of the mass distribution. Since it is only the mass distribution which is robustly predicted from theoretical models, it is very attractive to pursue methods which measure this distribution rather directly, without having to make assumptions about where galaxies should form in a given theoretical mass distribution.

Gravitational lensing is now established as a powerful method to measure directly the distribution of mass in the universe (e.g. Gunn 1967; Bartel- 
mann \& Schneider 1999 and references therein). This method is based on the measurement of the coherent distortions that lensing induces on the observed shapes of background galaxies. Recently, several groups have reported the statistical detection of weak lensing by large-scale structure (Wittman et al. 2000; van Waerbeke et al. 2000; Bacon, Refregier \& Ellis 2000; Kaiser, Wilson \& Luppino 2000). These detections offer remarkable prospects for precise measurements of the mass power spectrum and of cosmological parameters (e.g. Hu \& Tegmark 1998).

An assumption which is made in the interpretation of alignments in galaxy images is that the directions of projected images are intrinsically uncorrelated. At first sight, this seems a reasonable assumption to make; the redshift distribution of the background galaxies is usually very broad, so most source galaxies are not physically close. However, the signal which is sought in weak lensing studies is very small, with typical ellipticity correlations of $\sim 10^{-4}$. It is by no means obvious that the intrinsic correlations will be small enough to be negligible for lensing studies.

There are sound reasons for expecting some correlations in galaxy shapes. The shapes may be determined in part by the tidal gravitational field, which will have correlations at some level, or by correlation of torques during linear evolution. Shapes may also be influenced by merger events, which may not be isotropically distributed, but influenced by filamentary or other structures.

The realisation that intrinsic galaxy alignments might be important was made by a number of groups simultaneously, resulting in a number of independent studies (Heavens, Refregier \& Heymans 2000, Pen, Lee \& Seljak 2000, Croft \& Metzler 2000, Crittenden, Natarajan, Pen \& Theuns 2000, Catalan, Kamionkowski \& Blandford 2001). It should be said that a calculation of intrinsic shape correlations is by no means trivial. Analytically, the problem is very difficult; numerically, it is hard to get sufficient signal-to-noise; finally, it is plausible that shapes and their correlations are at least partly determined by non-gravitational processes which are difficult to model. In summary we are far from being able to predict robustly the ellipticity distribution of galaxies.

In order to make progress, the studies have made very different simplifying assumptions in relating the density field to the ellipticities of galaxies. The remarkable outcome is, however, a level of agreement of the level of the effect which one would perhaps not have expected in advance. The conclusion of all the studies is that for a broad distribution of sources centred around $z=1$, the alignment intrinsic in the galaxy distribution is a small but not insignificant contributor to the total expected from lensing and intrinsic effects. For a low-redshift $(z \simeq 0.1)$ source population, where the intrinsic effects are larger and the lensing effect smaller, the alignment of images is dominated almost 


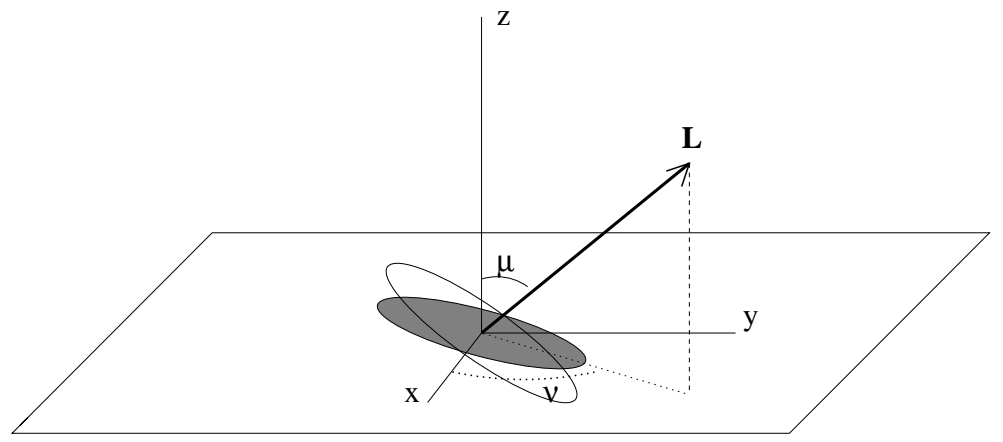

Figure 1: Simple model of a spiral galaxy. The disk of the galaxy is shown as the open ellipse, and its projection on the sky as the filled ellipse.

completely by intrinsic effects.

\section{Assumptions: galaxy ellipticity}

Catelan et al (2000) assumed that the shape is determined by the linear tidal field; Mackey et al. (2001) extended this model to consider disk galaxies in more detail. Lee, Pen \& Seljak (2000) and Crittenden et al. (2000) used a correlation, seen in N-body simulations between the tidal field and the moment of inertia tensor (Lee \& Pen 2000). From this they determined the angular momentum vector distribution. Numerical simulations from the VIRGO consortium were analysed by Heavens et al (2000) and Croft \& Metzler (2000), with similar conclusions. Both groups investigated an 'elliptical' model, where the ellipticity of the visible galaxy and the halo were assumed equal. Heavens et al. also looked a 'spiral' model, assuming that the galaxy was a thin disk with angular momentum vector aligned with that of the halo. Given that one is seeking a very small correlation of ellipticities, it would not be very surprising if these different assumptions led to rather different answers. This turns out not to be the case.

\section{Numerical simulations}

I will describe here the two approaches which use numerical simulations to estimate galaxy ellipticity correlations. The articles by Rob Crittenden and Jonathan Mackey in this volume will concentrate on more analytic approaches. Both numerical papers use outputs from Virgo simulations of 17 million particles, from which haloes are extracted using a friends-of-friends algorithm. 


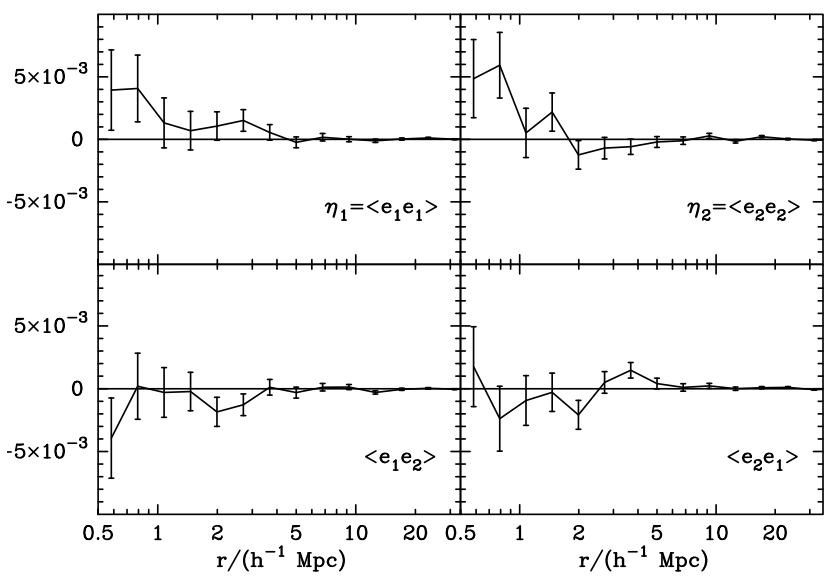

Figure 2: Three-dimensional correlation functions for 'spirals' in a $\Lambda C D M$ model at a redshift $z=1$.

Ellipticities in the spiral model are determined by the orientation of the angular momentum vector of the halo $\mathbf{L}$ with the line of sight (see fig. 11). With the coordinate system shown in the figure, the ellipticity $e \equiv\left(e_{1}^{2}+e_{2}^{2}\right)^{\frac{1}{2}}$ and position angle $\alpha$ of the projected ellipse are given by

$$
\begin{aligned}
e & =\frac{\sin ^{2} \mu}{1+\cos ^{2} \mu}, \\
\alpha & =\nu+\frac{\pi}{2} .
\end{aligned}
$$

and the ellipticity is $e_{i}=e\{\cos 2 \alpha, \sin 2 \alpha\}$. Note that the observed ellipticity depends only on the orientation of $\mathbf{L}$, and not on its magnitude; it is also independent of the surface brightness profile, provided it depends only on radius. In some sense, $e$ should be taken as an extreme: the average ellipticity of a randomly-oriented distribution of spirals is 0.57 - considerably larger than that observed. The ellipticity of the 'elliptical' models are calculated from halo quadrupole moments (Kaiser \& Squires 1993). In practice, the coordinate system is rotated so that the $x$-axis lies along the projected separation of the galaxy pairs.

It turns out that the ellipticities based on the spiral and elliptical prescriptions are very different. The ellipticity correlation functions, however, are rather similar (fig. 3 and 2). The ellipticities themselves are typically not 


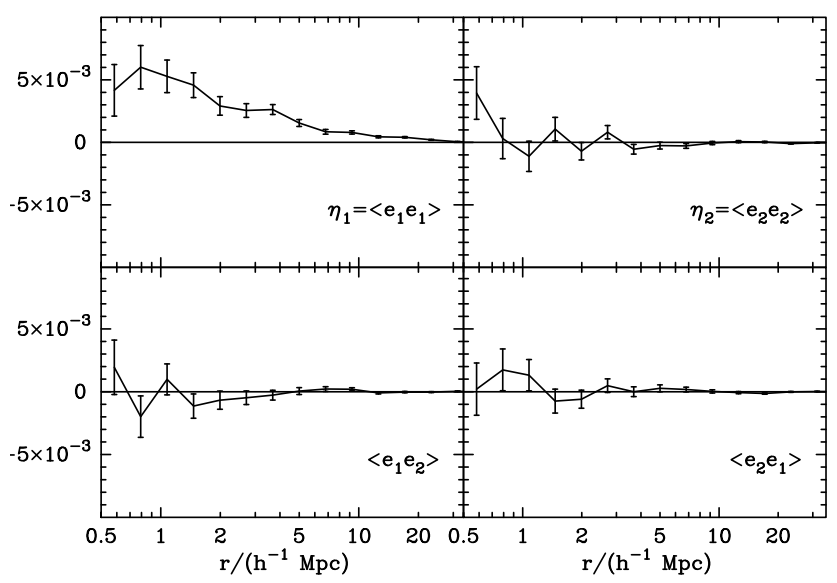

Figure 3: As fig. 2, but for 'ellipticals'.

much less than unity, so the correlation level of $\lesssim 5 \times 10^{-3}$ is rather small; the agreement, therefore, is by no means expected.

To assess the impact on the lensing signal, the three-dimensional correlation functions are turned into two-dimensional correlations, using a modified form of Limber's equation. Fig. A is typical of the results obtained for a deep survey at median redshift of unity. We see that the expected weak lensing signal (dotted) dominates, but the intrinsic signal is not entirely negligible, contributing $\sim 10 \%$ of the correlation. This general conclusion has been reached by all studies.

At low redshift, the story is quite different. The lensing signal is lower as there is less mass to pass through, and the intrinsic signal is larger, because fixed angular separations translate to smaller typical physical separations. For median redshifts of order unity, as expected in SuperCOSMOS and Sloan (Gunn et al 1995), the intrinsic effect dominates the lensing correlation by up to several orders of magnitude. This is illustrated in fig. 5, which shows the measured shear variance (which is related to the ellipticity correlation function) from the SuperCOSMOS survey, along with predictions from the various intrinsic studies (see also Pen, Lee \& Seljak 2000). Although there are certainly some significant differences between the studies, all agree that on small scales the intrinsic signal should be $\sim 10^{-2}$. 


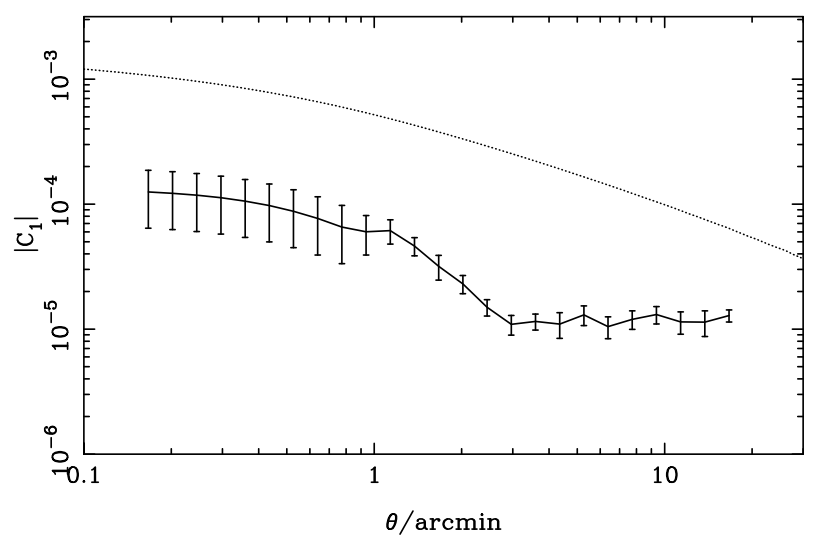

Figure 4: Intrinsic ellipticity correlation function of 'ellipticals', for a deep survey with median source redshift of 1 , and a broad source distribution. Also shown dotted is the expected weak lensing correlation signal. We see that the lensing signal dominates for this redshift, and intrinsic effects act as a $\sim 10 \%$ contaminant.

\section{Discussion}

I have summarised here the state of the investigations into the level of intrinsic alignment of galaxies, with a view to assessing its impact on weak gravitational lensing studies. Despite the difficulties of computing the correlation of ellipticities with confidence, several independent studies have come up with broadly similar conclusions. These are that the level of correlation is unlikely to be low enough to be ignored. For high-redshift $(z \simeq 1)$ lensing samples, the intrinsic correlation, being diluted over a wide redshift range, is a small, but non-negligible $(\sim 10 \%)$ fraction of the correlation induced by weak lensing. At low redshifts $(z \simeq 0.1)$, however, the lensing signal is smaller and the intrinsic correlation larger, to the extent that the intrinsic correlation dominates the lensing signal. This is also supported by observed correlations of galaxy shapes from the SuperCOSMOS survey (Brown et al. 2001), which shows distortions at a level far above the expected signal but broadly in line with theoretical predictions. There are some differences in the predictions for intrinsic correlations, but all agree with the above statements. It is perhaps difficult to see where the theoretical predictions could be made significantly more robust; certainly the methods based on numerical simulations would benefit by larger number of particles in each halo, and by larger volumes to improve signal-to- 


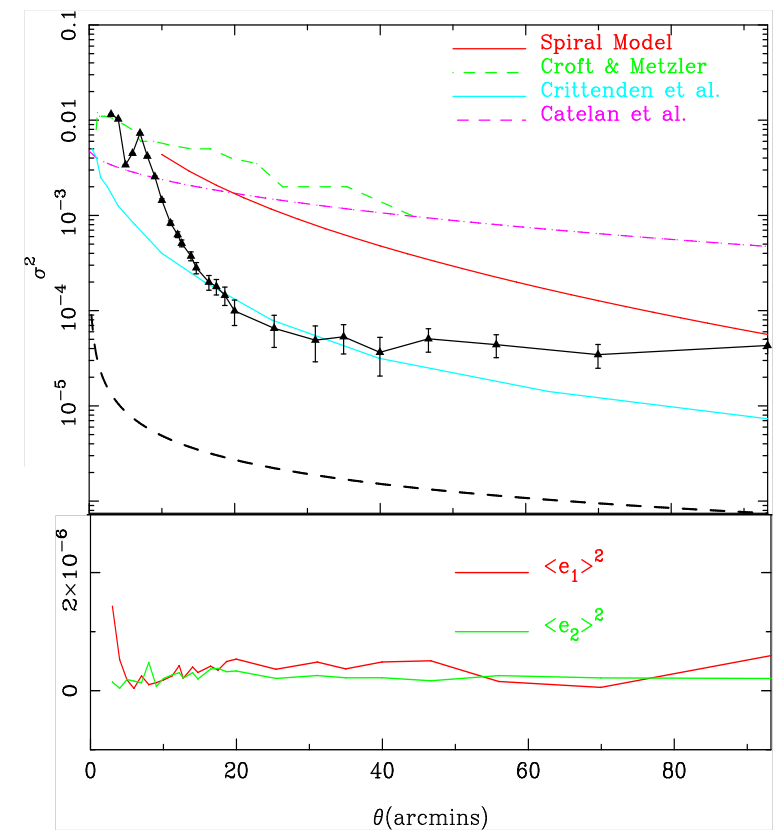

Figure 5: Shear variance estimate from the SuperCOSMOS survey (Brown et al 2001), compared with the 'spiral' model of Heavens et al (dark solid line), the elliptical model of Croft \& Metzler (upper dashed), the semi-analytic model of Crittenden et al (fainter solid), and the tidal stretch model of Catelan et al (lower dot-dashed). The expected lensing signal is the lowest line.

noise. Analytic methods are likely to be limited by the validity or otherwise of the simplifying assumptions, and neither general method can really be expected to include non-gravitational processes reliably in the near future. Thus it is probably best to be satisfied that we know the magnitude of the effect, and therefore not attempt to do straightforward weak lensing calculations from low-redshift samples. One can try to eliminate partly the intrinsic correlation by performing a curl-gradient decomposition of the ellipticity field, since weak lensing induces only a gradient field (Crittenden et al 2001, but see Mackey et al 2001). Alternatively, one can sidestep the problem almost entirely; this can be done, albeit by sacrificing some signal-to-noise, by cross-correlating orientations of galaxies which are known to be distant physically, for example by using photometric redshift information. 


\section{Acknowledgments}

I am grateful to my collaborators, Alexandre Refregier and Catherine Heymans, and also to Andy Taylor for the figures from the SuperCOSMOS survey. The simulations analysed in this paper were carried out using data made available by the Virgo Supercomputing Consortium (http://star-www.dur.ac.uk/ frazerp/virgo/) using computers based at the Computing Centre of the Max-Planck Society in Garching and at the Edinburgh Parallel Computing Centre. We are very grateful to Rob Smith for providing halos from the simulations.

\section{References}

1. Bacon D., Refregier A., \& Ellis R. 2000, submitted to MNRAS, preprint astro-ph/0003008

2. Bartelmann M., \& Schneider P. 1999, submitted to Physics Reports, preprint astro-ph/9912508

3. Brown M., Taylor A.N., Hambly N., Dye S., MNRAS, in press astro$\mathrm{ph} / 0009499$ )

4. Catelan P., Kamionkowski M., Blandford R.D., 2001, MNRAS, 320, 7

5. Crittenden R., Natarajan P., Pen U., \& Theuns T., 2000, submitted to ApJ, astroph/0009052

6. Crittenden R., Natarajan P., Pen U., \& Theuns T., 2001, submitted to ApJ, astro-ph/0012336

7. Croft R.A.C., Metzler C.A., 2000, ApJ, 545, 561

8. Heavens A.F., Refregier A., Heymans C.E.C., MNRAS, 319, 649

9. Gunn J.E., 1967, ApJ, 150, 737

10. Gunn J.E., Weinberg D.H., 1995, in Maddox S., Aragon-Salamanca A., eds., in Wide Field Spectroscopy and the Distant Universe, the $35^{\text {th }}$ Herstmonceux Conference", p.3

11. Hu W. \& Tegmark M. 1998 (astro-ph/9811168)

12. Kaiser N. \& Squires G. 1993, ApJ 404, 441.

13. Kaiser N., Wilson G., \& Luppino G.A., 2000, submitted to ApJL, preprint astro-ph/0003338

14. Lee J., Pen U.-L., 2000, ApJ, 532, 5

15. Pen U.-L., Lee J., Seljak U., 2000, ApJ, 543, L107

16. Rhodes J., Refregier A., \& Groth E., 1999, to appear in ApJ, astro$\mathrm{ph} / 9905090$

17. Van Waerbeke L., et al. 2000, submitted to A\&A, preprint astro$\mathrm{ph} / 0002500$

18. Wittman D.M., Tyson J.A., Kirkman D., Dell'Antonio I., \& Bernstein G., 2000, submitted to Nature, preprint astro-ph/0003014 